\title{
EPITAXIALLY-GROWN GALLIUM NITRIDE ON GALLIUM OXIDE SUBSTRATE FOR PHOTON PAIR GENERATION IN VISIBLE AND TELECOMM WAVELENGTHS
}

\author{
Kashif M. Awan and Ksenia Dolgaleva \\ School of Electrical Engineering and Computer Science \\ Department of Physics and Max Planck Centre for \\ Extreme and Quantum Photonics \\ University of Ottawa \\ Ottawa, Canada \\ Ksenia.Dolgaleva@uottawa.ca
}

\author{
Mufasila M. Muhammed and Iman S. Roqan \\ Physical Sciences and Engineering Division \\ King Abdullah University of Science and Technology \\ Thuwal, Saudi Arabia \\ iman.roqan@kaust.edu.sa
}

\begin{abstract}
Keywords - nonlinear optics; integrated photonic circuits; four-wave mixing; all-optical wavelength conversion; photon pair generation; GaN epitaxial growth
\end{abstract}

Gallium Nitride (GaN), along with other III-Nitrides, is attractive for optoelectronic and electronic applications due to its wide direct energy bandgap, as well as high thermal stability. $\mathrm{GaN}$ is transparent over a wide wavelength range from infra-red to the visible band, which makes it suitable for lasers and LEDs. It is also expected to be a suitable candidate for integrated nonlinear photonic circuits for a wide range of applications from all-optical signal processing to quantum computing and on-chip wavelength conversion. Despite its abundant use in commercial devices, there is still need for suitable substrate materials to reduce high densities of threading dislocations (TDs) and other structural defects like stacking faults, and grain boundaries. All these defects degrade the optical quality of the epi-grown GaN layer as they act as non-radiative recombination centers.

$\mathrm{GaN}$ is commonly grown on transparent Sapphire $\left(\mathrm{Al}_{2} \mathrm{O}_{3}\right)$ as well as conductive Silicon Carbide $(\mathrm{SiC})$ substrates. The former, however, suffers from lattice mismatch of $14 \%$, leading to higher density of TDs [1] and, while the latter is expensive but lacks transparency in the visible range due to doping [2]. Studies have shown that GaN grown on (-201) $\beta$ Gallium Oxide $\left(\mathrm{Ga}_{2} \mathrm{O}_{3}\right)$ has superior optical quality due to a better lattice matching. In addition, it is also a conductive, thermally stable and transparent substrate $[3,4]$. In this work, we propose $\mathrm{GaN}$ epitaxially grown on $(-201) \beta-\mathrm{Ga}_{2} \mathrm{O}_{3}$ as a suitable candidate for correlated photon pair generation, leading to on-chip quantum sources for both telecomm and visible spectrum. We also present designs for $\mathrm{GaN}$ waveguides to achieve efficient four-wave mixing (FWM) based on the experimental absorption and dispersion data of epitaxially grown $\mathrm{GaN}$ on $\mathrm{Ga}_{2} \mathrm{O}_{3}$.

We also present a detailed modal and dispersion analysis of $\mathrm{GaN}$ waveguide designs for enhanced optical nonlinear interactions, resulting in efficient FWM. These waveguides can later be used as on-chip sources for correlated photon pair generation using spontaneous FWM. Such sources could be realized in both simple straight waveguides and microring resonators $[5,6]$. We present two separate waveguide designs: one for the visible spectrum, and another one for the telecom C-band. Both designs are optimized for the modal confinement, as well as dispersion and losses induced due to fabrication tolerances [7]. With the improvement in the optical quality, offered by the growth on the lattice-matched $\mathrm{Ga}_{2} \mathrm{O}_{3}$ substrate, and the optimization of the waveguide geometry to enhance FWM efficiency, we believe that this study can be a stepping stone toward realizing the first on-chip source of correlated photon pairs in the visible spectral range.

\section{REFERENCES}

[1] N. V. Edwards, et al., III-Nitride Semiconductors: Electrical, Structural and Defects Properties , pp. 287-337 (2000).

[2] L. Liu, et al., Mater. Sci. Eng., R 37(3), 61-127 (2002).

[3] M. M. Muhammed, et al., Applied Physics Letters 105, 042112 (2014).

[4] K. Shimamura, et al., Japanese Journal of Applied Physics 44, L7-L8 (2005).

[5] Jay E. Sharping, et al., Opt. Express 14, 12388-12393 (2006).

[6] S. Clemmen, et al., Optics Express 17, 16558-16570 (2009).

[7] K. M. Awan, et al., Proc. Photonics North 2015, pp.1, 9-11. 